\title{
Comparative transcriptomics of choroid plexus in Alzheimer's disease, frontotemporal dementia and Huntington's disease: implications for CSF homeostasis
}

Edward G. Stopa ${ }^{1 \dagger},{\text { Keith Q. } \text { Tanis }^{2 \dagger} \text {, Miles C. Miller }}^{1}$, Elena V. Nikonova ${ }^{2}$, Alexei A. Podtelezhnikov², Eva M. Finney², David J. Stone², Luiz M. Camargo², Lisan Parker², Ajay Verma ${ }^{3}$, Andrew Baird ${ }^{4}$, John E. Donahue', Tara Torabi', Brian P. Eliceiri ${ }^{4}$, Gerald D. Silverberg ${ }^{1}$ and Conrad E. Johanson ${ }^{1 *}$

\begin{abstract}
Background: In Alzheimer's disease, there are striking changes in CSF composition that relate to altered choroid plexus (CP) function. Studying CP tissue gene expression at the blood-cerebrospinal fluid barrier could provide further insight into the epithelial and stromal responses to neurodegenerative disease states.

Methods: Transcriptome-wide Affymetrix microarrays were used to determine disease-related changes in gene expression in human CP. RNA from post-mortem samples of the entire lateral ventricular choroid plexus was extracted from 6 healthy controls (Ctrl), 7 patients with advanced (Braak and Braak stage III-VI) Alzheimer's disease (AD), 4 with frontotemporal dementia (FTD) and 3 with Huntington's disease (HuD). Statistics and agglomerative clustering were accomplished with MathWorks, MatLab; and gene set annotations by comparing input sets to GeneGo (http://www. genego.com) and Ingenuity (http://www.ingenuity.com) pathway sets. Bonferroni-corrected hypergeometric p-values of $<0.1$ were considered a significant overlap between sets.
\end{abstract}

Results: Pronounced differences in gene expression occurred in CP of advanced AD patients vs. Ctrls. Metabolic and immune-related pathways including acute phase response, cytokine, cell adhesion, interferons, and JAK-STAT as well as mTOR were significantly enriched among the genes upregulated. Methionine degradation, claudin- 5 and protein translation genes were downregulated. Many gene expression changes in AD patients were observed in FTD and HuD (e.g., claudin-5, tight junction downregulation), but there were significant differences between the disease groups. In $A D$ and $H u D$ (but not FTD), several neuroimmune-modulating interferons were significantly enriched (e.g., in AD: IFI-TM1, IFN-AR1, IFN-AR2, and IFN-GR2). AD-associated expression changes, but not those in HuD and FTD, were enriched for upregulation of VEGF signaling and immune response proteins, e.g., interleukins. HuD and FTD patients distinctively displayed upregulated cadherin-mediated adhesion.

Conclusions: Our transcript data for human CP tissue provides genomic and mechanistic insight for differential expression in AD vs. FTD vs. HuD for stromal as well as epithelial components. These choroidal transcriptome characterizations elucidate immune activation, tissue functional resiliency, and CSF metabolic homeostasis. The BCSFB undergoes harmful, but also important functional and adaptive changes in neurodegenerative diseases; accordingly,

\footnotetext{
*Correspondence: Conrad_Johanson@brown.edu

${ }^{\dagger}$ Edward G. Stopa and Keith Q. Tanis contributed equally to this work

${ }^{1}$ Departments of Neurosurgery and Pathology (Neuropathology

Division), Rhode Island Hospital, The Warren Alpert Medical School, Brown

University, Providence, RI, USA

Full list of author information is available at the end of the article
}

(c) The Author(s) 2018. This article is distributed under the terms of the Creative Commons Attribution 4.0 International License (http://creativecommons.org/licenses/by/4.0/), which permits unrestricted use, distribution, and reproduction in any medium, provided you give appropriate credit to the original author(s) and the source, provide a link to the Creative Commons license, and indicate if changes were made. The Creative Commons Public Domain Dedication waiver (http://creativecommons.org/ publicdomain/zero/1.0/) applies to the data made available in this article, unless otherwise stated. 
the enriched JAK-STAT and mTOR pathways, respectively, likely help the CP in adaptive transcription and epithelial repair and/or replacement when harmed by neurodegeneration pathophysiology. We anticipate that these precise CP translational data will facilitate pharmacologic/transgenic therapies to alleviate dementia.

Keywords: Choroid plexus transcriptome, Neuroimmune CSF regulation, Blood-CSF barrier inflammatome, Janus kinase/signal transducers and activators of transcription (JAK-STAT), Peroxisome-proliferator-activated receptor (PPAR), Cadherin-mediated adhesion, Vascular endothelial growth factor, LRP-1, Choroid plexus methionine, CSF homocysteine, Mechanistic target of rapamycin (mTOR)

\section{Background}

The choroid plexus (CP) is a CNS secretory tissue within the cerebroventricular system consisting of a vascular stroma surrounded by epithelium [1,2]. Although the primary function of choroidal tissue is to produce and regulate cerebrospinal fluid (CSF), it also importantly provides a permeability-regulating blood-CSF barrier (BCSFB) [3]. Other additional roles of CP relate to CNS wound repair [4], sex hormone modulation of BCSFBCNS [5], catabolite detoxification [6], ion regulation [7], a selective leukocyte gate [8], and CSF-brain neuroimmune homeostasis, including interferon actions [9-13]. Recently, the BCSFB tissue has been examined for unique $\mathrm{CP}$ changes in diverse disorders: mitochondrial diseases [14], multiple sclerosis/experimental autoimmune encephalitis [15, 16], schizophrenia [17], acute response to peripheral immune challenge [18], normal pressure hydrocephalus [19], and Alzheimer's disease (AD) [20, 21].

Analyzing the transformed CP tissue composition and pathophysiologic functions in neurodegeneration elucidates specific metabolic/secretory processes underlying CSF-CNS disease pathogenesis [22]. BCSFB alterations such as choroid epithelial cell atrophy, stromal fibrosis, vascular thickening, tight junction (claudin-5) downregulation, and basement membrane thickening are associated with $\mathrm{AD}$ pathology [20]. These changes in the epithelial-stromal nexus likely affect secretion and transport, resulting in diminished CSF turnover and modified neuroimmune regulation. Neuroimmune phenomena in the CP and/or CSF include adjustments in the level of proteins (e.g., neurotrophins, interferons and growth factors), cytokines, and certain immune cells [12, 22]. Oxidative stressors in AD and other dementias may also differentially impact CP's ability to synthesize/transport proteins/hormones, and to regulate cellular/CSF metabolites such as methionine/homocysteine [23], $\mathrm{A} \beta / \operatorname{tau}$ [24], and creatine/creatinine [25].

This investigation at the Brown University Medical School, in collaboration with Merck \& Co., analyzed gene expression in $\mathrm{CP}$ tissues from late-stage Alzheimer patients, for comparison with control subjects (Ctrl) and two other diseases: frontotemporal dementia (FTD) and Huntington's disease $(\mathrm{HuD})$. Our working hypothesis anticipated: (i) common denominators of altered $\mathrm{CP}$ expression in the three diseases, as well as (ii) differential expression patterns due to disease-specific alterations in neural metabolites, that by 'homeostatic feedback signaling' via volume transmission from brain to CSF to CP, could uniquely modulate gene expression at the BCSFB.

Investigating $\mathrm{CP}$ tissue gene expression in various $\mathrm{CNS}$ diseases likely informs on diverse BCSFB adjustments to neurodegeneration. Bergen et al. [26] focused on gene expression changes by $\mathrm{CP}$ epithelial cells in AD. In this study, we analyze CP tissue responses (epithelium plus stroma) by providing profiles of mRNA changes. The altered expression profiles in $\mathrm{AD}, \mathrm{FTD}$ and $\mathrm{HuD}$ are discussed in relation to restorative homeostatic mechanisms, as well as to chronic BCSFB damage and disrupted CSF-brain homeostasis.

\section{Methods}

Project approval, sample collection and demographics

This research with banked specimens of human CP tissue was approved by the Institutional Review Board for Clinical Research at Lifespan, Rhode Island Hospital, Providence, RI. Post-mortem tissue samples from 6 healthy Ctrls of mean age 60 years, mean post-mortem interval (PMI) $22 \mathrm{~h}$; and from 7 patients with advanced $\mathrm{AD}$ (Braak and Braak stage III-VI, 80 years, PMI $17 \mathrm{~h}$ ), 4 FTD (72 years, PMI NA) and $3 \mathrm{HuD}$ (71 years, PMI $19 \mathrm{~h}$ ), were snap frozen in liquid $\mathrm{N}_{2}$ and stored at $-80^{\circ} \mathrm{C}$ in the Brown University Brain Tissue for Neurodegenerative Disorders Resource Center until processed. Demographic and disease data for the individual controls and patients are presented in Table 1.

\section{Microarray design and analysis}

RNA from CP was extracted using TRIzol reagent in accordance with instructions by manufacturer (ThermoFisher, Grand Island, NY). Isolated RNA samples were assayed for quality via the Agilent RNA 6000 Pico Kit on Agilent Bioanalyzer (Santa Clara, CA) and RNA yield via Quanti-iT RiboGreen RNA Assay Kit (ThermoFisher). Samples were amplified and labeled using an automated version of the NuGEN Ovation WB protocol 
Table 1 Demographic and clinical data of choroid plexus samples collected

\begin{tabular}{lllll}
\hline Sample ID \# & Diagnosis & Age & Sex & PMI \\
\hline CP_CTR_007 & Control & 62 & $\mathrm{M}$ & 23.9 \\
CP_CTR_008 & Control & 55 & $\mathrm{~F}$ & 29 \\
CP_CTR_009 & Control & 37 & $\mathrm{M}$ & 18.7 \\
CP_CTR_010 & Control & 64 & $\mathrm{M}$ & 25.8 \\
CP_CTR_011 & Control & 69 & $\mathrm{~F}$ & 12.3 \\
CP_CTR_012 & Control & 70 & $\mathrm{M}$ & $\mathrm{N} / \mathrm{A}$ \\
CP_ALZ_015 & AD (Braak III-IV) & 74 & $\mathrm{~F}$ & 15 \\
CP_ALZ_017 & AD (severe BraakV-VI) & 84 & $\mathrm{~F}$ & $\mathrm{~N} / \mathrm{A}$ \\
CP_ALZ_018 & AD (severe BraakV-VI) & 84 & $\mathrm{M}$ & $\mathrm{N} / \mathrm{A}$ \\
CP_ALZ_019 & AD (severe + Lewy body disease) & 84 & $\mathrm{~F}$ & $\mathrm{~N} / \mathrm{A}$ \\
CP_ALZ_020 & AD (severe BraakV-VI) & 89 & $\mathrm{M}$ & $\mathrm{N} / \mathrm{A}$ \\
CP_ALZ_022 & AD (severe BraakV-VI) & 73 & $\mathrm{M}$ & 24.5 \\
CP_ALZ_023 & AD (severe BraakV-VI) & 70 & $\mathrm{M}$ & 10.8 \\
CP_FTD_024 & FTD & 76 & $\mathrm{M}$ & $\mathrm{N} / \mathrm{A}$ \\
CP_FTD_025 & FTD and motor neuron disease & 75 & $\mathrm{~F}$ & $\mathrm{~N} / \mathrm{A}$ \\
CP_FTD_026 & FTD Pick's disease & 58 & $\mathrm{M}$ & $\mathrm{N} / \mathrm{A}$ \\
CP_FTD_027 & FTD & 80 & $\mathrm{~F}$ & $\mathrm{~N} / \mathrm{A}$ \\
CP_HuD_029 & HuD (grade IV) & 68 & $\mathrm{M}$ & 30.1 \\
CP_HuD_030 & HuD (grade IV) & 65 & $\mathrm{~F}$ & 3.5 \\
CP_HuD_031 & HuD (grade IV) & 80 & $\mathrm{M}$ & 24 \\
\hline
\end{tabular}

after normalizing to $50 \mathrm{ng}$ total RNA input (NuGEN Technologies, San Carlos, CA). Gene expression profiling was performed with a customized Human Affymetrix GeneChip microarray (GEO platform GPL 10379) that included 57,060 probe sets (Affymetrix, Santa Clara, CA). Hybridization $\left(45^{\circ} \mathrm{C}\right.$ for $18 \mathrm{~h}$ ), labeling, and scanning, using Affymetrix ovens, fluidics stations, and scanners, were conducted following the protocols recommended (NuGEN Technologies). All 20 samples passed RNA integrity and Affymetrix quality control metrics. The final sample set contained RNA from 6 Ctrl, $7 \mathrm{AD}$, 4 FTD and $3 \mathrm{HuD}$ subjects (Table 1, Additional file 1: Table S1).

\section{Data processing, statistics and annotation}

Data were normalized by robust multiarray average (RMA) [27], and each sample was ratioed to the average of the Ctrl samples [28]. Statistical analysis and agglomerative clustering were performed using MathWorks MatLab (Natick, MA). In some statistical analyses, due to insufficient power, $\mathrm{HuD}$ and FTD data were combined into one non-AD disease grouping, as indicated by: (HuD +FTD). Gene set annotation analysis was performed by comparing input sets to GeneGo (http://www. genego.com) and Ingenuity (http://www.ingenuity.com) pathway sets. Bonferroni-corrected hypergeometric p-values (expectation (e)-values) of $<0.1$ were considered a significant overlap between sets.

\section{Results}

We first compared the genome-wide differences in gene expression between diseased and Ctrl CP. p-value distributions from T-test comparisons, between the Ctrl group and each of the neurodegenerative disease groups (AD, $\mathrm{HuD}, \mathrm{FTD}$ ), revealed significant effects on the CP transcriptome in each of the 3 diseases (Fig. 1a, Additional file 2: Table S2). The AD group had the highest number of differentially expressed probe sets likely due, at least in part, to the higher number of $\mathrm{AD}$ subjects compared to $\mathrm{HuD}$ and FTD. 3935 (7\%) out of the 57,060 probe sets on the array were differentially expressed $[p<0.01$, false discovery rate $(\mathrm{FDR})=14 \%$ ] between $\mathrm{AD}$ and Ctrl subjects, while $1287(\mathrm{FDR}=44 \%)$ and $2136(\mathrm{FDR}=27 \%)$ probe sets were regulated with $\mathrm{p}<0.01$ in $\mathrm{HuD}$ and FTD, respectively (Fig. 1a, b, Additional file 2: Table S2). Despite the limited statistical power and resulting high false discovery rates in the $\mathrm{HuD}$ and FTD comparisons, there was a large degree of overlap in the genes identified in each of the comparisons (Fig. 1b, c, Additional file 2: Table S2). Almost all probe sets differentially expressed $(\mathrm{p}<0.01)$ in the AD samples had significant or a trend toward differential expression in the same direction in the other two disease groups (Fig. 1c, Additional file 2: Table S2). In Additional file 4: Table S4 are listed the top 10 most upregulated and 10 most downregulated genes from the $\mathrm{AD}$ vs. Ctrl comparison; $80 \%$ of these findings were confirmed by multiple probe sets when available on the array.

In order to validate these findings with different subjects and gene expression platforms, we compared the results in whole $\mathrm{CP}$ to those obtained by Bergen et al. in laser-dissected CP epithelial cells from control and $\mathrm{AD}$ subjects [26]. We anticipated that the changes reported in $\mathrm{CP}$ epithelial cells would also be evident in the whole CP samples but to a lesser degree given the presence of additional cell types such as stroma and immune cells in the whole tissue samples. Indeed, the 36 genes reported as differentially expressed in AD CP epithelium by microarray, and in some cases [17] also by quantitative PCR (Bergen et al. Tables 1 and 2 in [26]), were regulated similarly in $\mathrm{AD}$ whole $\mathrm{CP}$ tissue in our study $\left(\mathrm{r}^{2} \sim 0.7\right)$, but to a lesser magnitude (by $~ 35 \%$, as indicated by linear regression in Fig. 1d). 34 of the 36 differentially expressed genes reported by Bergen et al. [26] were modulated in the same direction from Ctrl in both studies, with 20 obtaining significance $(\mathrm{p}<0.05)$ in the current AD whole CP tissue comparison. Similarly the expression values in whole $\mathrm{CP}$ from FTD and $\mathrm{HuD}$ 

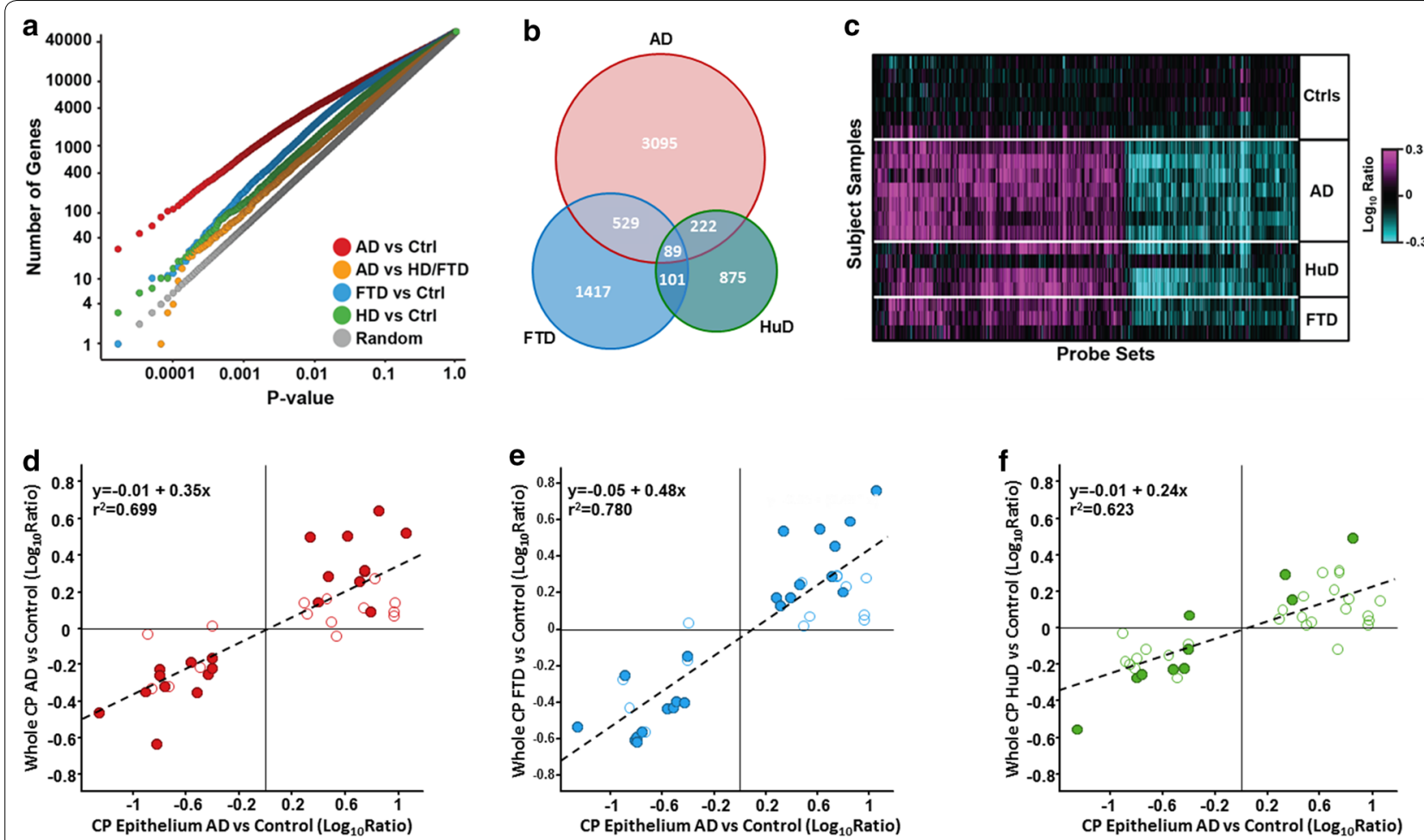

Fig. 1 Significant gene expression differences between Ctrl and diseased CP: a T-test p-value distributions among all probe sets for AD (red), FTD (blue) and HuD (green) vs. Ctrl samples, as well as AD vs. combined FTD plus HuD samples (orange). Gray data points indicate number of significant probe sets expected by chance. b Overlap of probe sets differentially expressed ( $p<0.01$ ) between Ctrl and AD (red), Ctrl and FTD (blue) and Ctrl and $\mathrm{HuD}$ (green) subjects. $\mathbf{c}$ Heatmap of probe sets differentially expressed $(p<0.01)$ between AD and Ctrl subjects. Probe sets were ordered by agglomerative clustering. Correlation between expression changes in whole CP from AD (d), FTD (e), and HuD (f) to those reported by Bergen et al. [26] in laser-dissected CP epithelial cells from AD subjects. Plotted are the 36 genes reported in Tables 1 and 2 by Bergen et al. [26] that were also represented on the array used in our study. Values are relative to corresponding study Ctrl subjects. Filled circles had $p<0.05$ in the corresponding whole CP comparisons. Dotted lines, the provided equation and $r^{2}$ values represent linear fit of the data

also correlated with those reported for AD CP epithelium in reference \# [26] $\left(\mathrm{r}^{2}=0.8\right.$ and 0.6 , respectively, Fig. 1e, f).

In this study, among the 3935 probe sets differentially expressed $(\mathrm{p}<0.01)$ in AD compared to Ctrl subjects, 2332 were upregulated and 1603 downregulated (Fig. 1b, Additional file 2: Table S2). The differentially expressed genes were examined for overlap with $\sim 2000$ GeneGo and Ingenuity pathways. Ninety-two pathways were enriched (Bonferroni corrected p-value, i.e., e-value, $<0.01)$ among the upregulated genes. These enrichments represented primarily immune-related pathways, including acute phase response, cytokine and interferon signaling, NFkB, and cell adhesion, as well as growth factor, JAK-STAT and mTOR signaling pathways, PPAR signaling and protein/nucleic acid salvage pathways (Fig. 2, Additional file 3: Table S3a). Pathway enrichment among downregulated genes was less extensive (12 pathways with $\mathrm{e}<0.01$ ), including genes involved in methionine degradation and protein translation (Fig. 2, Additional file 3: Table S3b).
Many differences between $\mathrm{AD}$ and Ctrl were also observed in $\mathrm{HuD}$ vs. FTD (Fig. 1, Additional file 2: Table S2). However, there were also some significant differences between AD vs. HuD + FTD, with 902 (1.6\%) probe sets significant at $\mathrm{p}<0.01$ (63\% FDR) (Fig. 3). Figure 3a displays the genes up and down-regulated in $\mathrm{AD}$ more than in the combined $\mathrm{HuD}+\mathrm{FTD}$ group; whereas Fig. $3 \mathrm{~b}$ presents the opposite, i.e., genes regulated more in $\mathrm{HuD}+\mathrm{FTD}$ than in $\mathrm{AD}$. The 513 probe sets uniquely upregulated in $\mathrm{AD}$ : $\mathrm{AD}$ vs. Ctrl $(\mathrm{p}<0.01)$ and $\mathrm{AD}$ vs. $\mathrm{HuD}+$ FTD $(\mathrm{p}<0.05)$ were enriched $(\mathrm{e}<0.1)$ predominately in interleukin and VEGF signaling genes (Additional file 3: Table S3c). There were 272 probe sets uniquely downregulated in $\mathrm{AD}$ : $\mathrm{AD}$ vs. $\mathrm{Ctrl}(\mathrm{p}<0.01)$ and AD vs. HuD + FTD $(\mathrm{p}<0.05)$ but were not significantly $(\mathrm{e}<0.1)$ enriched in any queried pathway. The 112 probe sets uniquely upregulated in $\mathrm{HuD}+\mathrm{FTD}$, that is, $\mathrm{HuD}+\mathrm{FTD}$ vs. Ctrl $(\mathrm{p}<0.01)$, and $\mathrm{HuD}+\mathrm{FTD}$ vs. AD $(\mathrm{p}<0.05)$, were enriched $(\mathrm{e}<0.1)$ in cadherin-mediated cell adhesion (Additional file 3: Table S3d). The 115 probe sets uniquely downregulated in $\mathrm{AD}$ vs. Ctrl $(\mathrm{p}<0.01)$, 

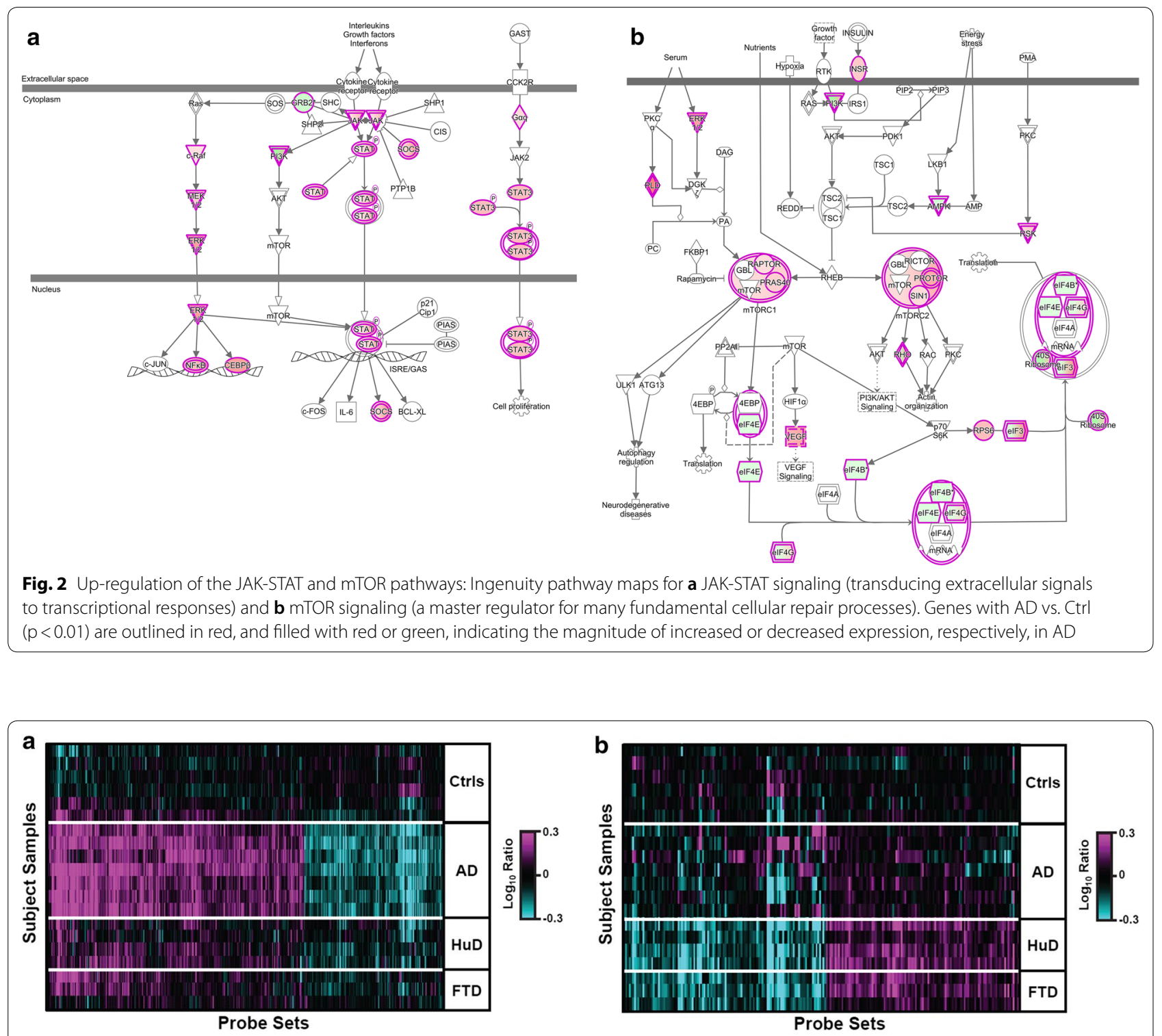

Fig. 3 Expression changes unique to AD, or to the combined HuD +FTD, non-AD 'disease control' group: a Heatmap of probe sets differentially expressed between $A D$ and $C$ trl subjects $(p<0.01)$, and altered more relative to the control in the AD group than in the non-AD'disease control' group $(\mathrm{AD} / \mathrm{Ctrl}) /(\mathrm{HuD}+\mathrm{FTD} / \mathrm{Ctrl})>1$, and AD vs. HuD + FTD, $(p<0.05)$. Probe sets are ordered by agglomerative clustering. $\mathbf{b}$ Heatmap of probe sets differentially expressed between the combined HuD + FTD group and Ctrl subjects $(p<0.01)$, and altered more relative to the combined $\mathrm{HuD}+\mathrm{FTD}$ disease control group than in the AD group ( HuD + FTD/Ctrl)/(AD/Ctrl) $>1$, and HuD + FTD vs. AD, $(p<0.05)$. Probe sets were ordered by agglomerative clustering. Red (magenta) and green (cyan) indicate the magnitude of increased and decreased expression, respectively

and $\mathrm{AD}$ vs. HuD + FTD $(\mathrm{p}<0.05)$, were not significantly $(\mathrm{e}<0.1)$ enriched in assessed biological pathways.

The emphasis here is on gene sets and associated biological pathways. Still, it is instructive to focus on several genes/proteins currently of great interest in CP pathophysiology. Altered tight junction protein claudin-5 in $\mathrm{CP}$ is associated with BCSFB breaching [26], while interferon has a protein-signaling role that couples choroidalcerebral neuroimmune interactions [13]. Claudin-5 was downregulated 1.6-, 1.9- and 2.5-fold in AD, FTD and $\mathrm{HuD}$, respectively, reaching significance in FTD (respective $p$ values of $0.071,0.028$ and 0.059). Multiple interferon signaling genes were upregulated in AD: IFI-TM1 $(p=0.0008)$, IFN-AR1 $(p=0.006)$, IFN-AR2 $(p=0.0007)$ and IFN-GR2 $(\mathrm{p}=0.0002)$. The complete lists of differentially-expressed genes, as well as enriched biological pathways, are provided in Additional file 2: Table S2, Additional file 3: Table S3, Additional file 4: Table S4. 


\section{Discussion}

Comparing gene expression in $\mathrm{CP}$ of $\mathrm{AD}$ subjects to that of Ctrls and other neurodegenerative diseases reveals important biological functions altered by dementia generation and progression. The $\mathrm{CP}$ as a dynamic interface between blood, CSF and brain, is able to monitor distortions and homeostatically respond, e.g., by the JAK-STAT pathway as well as cytokine and protein-signaling molecules [12, 13]. These homeostatic adjustments impact neural viability. This intimates that $\mathrm{CP}$ beneficial adjustments, as well as BCSFB malfunctioning, are pertinent to $\mathrm{AD}$ progression. The strategic role of CSF to safeguard brain is manifested by CP upregulation of many genes in response to neurodegeneration ([26] and this investigation); and by its ability to protect basic CSF composition (e.g., K, pH and vitamin homeostasis) even in advanced AD [7]. Nevertheless, the CP in AD incurs structural damage [20] and distorted epithelial metabolism and transport (e.g., A $\beta$, cytokine (e.g., TNF $\alpha$ ) and methionine/homocysteine [24, 29]). The injured epithelium likely depends on enhanced mTOR expression to facilitate cellular repair and/or replacement. Accordingly, the state of $\mathrm{CP}-\mathrm{CSF}$ viability in ongoing neurodegeneration is a balance between debilitating and restorative events at the BCSFB [29-31].

In this study we have shown numerous transcriptional alterations in the $\mathrm{CP}$ of subjects with neurodegenerative disease. Given that the BCSFB makes adjustments in CSF composition, the prolific disease-induced expression changes in $\mathrm{CP}$ fits previous homeostasis modeling [1, 30]. Most transcript changes were common to AD, FTD and $\mathrm{HuD}$, while fewer genes were modulated differently between $\mathrm{AD}$ and $\mathrm{HuD}+\mathrm{FTD}$. It is instructive to compare the present observations with an earlier transcriptional investigation of the BCSFB in AD [26]. Whereas we studied homogenates of entire $\mathrm{CP}$, including epithelium and stroma, Bergen et al. studied single epithelial cells captured by laser microscopy [26]. Thus, Bergen et al. provided data on epithelium-specific changes in AD (larger-fold changes, see Fig. 1d-f), while our dataset also includes potential pathophysiological interactions between CP stroma and epithelium. Indeed, robust inflammatory responses were not reported by Bergen et al. [26], suggesting deductively that the immune-reactive pathophysiology occurs primarily within CP stroma.

Some limitations of our study include the small number of patients in the HuD and FTD groups, and the lack of direct confirmation of specific genes by RT-PCR. Future studies with a larger $\mathrm{N}$ value for $\mathrm{HuD}$ and FTD will increase the statistical power for disease comparisons. For post-mortem tissue, RNA stability is challenging with autopsy specimens collected at various PMIs; however, we carefully assessed RNA integrity, using only samples passing stringent criteria. Moreover, our emphasis was on significantly-enriched pathways (affecting multiple genes within a given pathway) rather than specific gene targets, with possible individual false discoveries. Any residual blood elements in specimens would unlikely explain differences in tissue mRNA among the three disease groups. The somewhat younger Ctrl group may be advantageous in avoiding potentially confounding issues with clinically-silent early dementia in an older 'Ctrl' cohort, otherwise presumed normal.

We used GeneGo (http://www.genego.com) and Ingenuity (http://www.ingenuity.com) sets for pathway analysis. In $\mathrm{AD}$ compared to Ctrls, this revealed upregulated inflammation genes: acute phase response, cell adhesion and cytokine, interferon, JAK-STAT signaling (for translating extracellular signals into transcriptional responses). Notable downregulated pathways were methionine degradation and protein translation; both are implicated in AD pathology. Claudin-5 expression was downregulated, consistent with the enhanced leakiness of the BCSFB [31] encountered in neurodegenerative diseases [26] and pathophysiology models [29]. Amyloid beta peptide (A $\beta$ ) damages $\mathrm{CP}$ tight junctions by activating matrix metalloproteineases, thereby increasing paracellular permeability [32]. The functional significance of such altered CP pathways, for disease outcome, awaits elucidation.

Prominent in AD was upregulated inflammationrelated signaling. These inflammation signatures differ from cortex-associated microglial infiltration [33, 34]. Key marker genes of cortical inflammation-APOE, TREM2, TYROBP-did not upregulate in AD CP. Rather, acute phase response genes dominated the upregulation: multiple cytokine and interferon receptors, JAK-STAT signaling components, MAPK, NFKB signaling and cell adhesion. Cytokines and growth factors in disease-associated reactions in BCSFB come from brain [35], blood, or $\mathrm{CP}$ itself. The $\mathrm{CP}$ responds biochemically and transcriptionally to circulating cytokines, central injury and systemic diseases [18, 36, 37]. Localized CP immunoreactions (e.g., inflammation-resolving leukocyte trafficking) may benefit brain by sensing 'injury signals' flowing from brain to CSF to CP, then feeding back to make homeostatic neural adjustments [35].

At certain stages of advancing neuroinflammation (caused by brain-residing pro-inflammatory microglial responses to $A \beta$ loads), the $C P$ receives plasma interferon $\gamma$ as a signal to promote homeostatic transport of anti-inflammatory monocyte-derived macrophages into CSF for resolving parenchymal inflammation [12]. Disease-induced disruption of this neuroimmune interferon adjustment at the BCSFB [38] could compromise the ability of the CSF-brain to thwart AD exacerbation. The CP competently adapts to AD stress [39] 
by maintaining an immunosuppressant profile of factors, e.g., VEGF and TGF $\beta 1$ in CSF, to help manage brain inflammation after neuronal injury [40].

Increased expression of LRP- 1 , a choroidal $A \beta$ transporter, agrees with mouse AD modeling [24]. Upregulated LRP-1 in the apical membrane expedites A $\beta$ removal from CSF [24]. Augmented reabsorptive clearance of $A \beta$ at the BCSFB aids the CNS because cerebral capillaries in AD extrude less $A \beta$ [41]. This compensatory $\mathrm{A} \beta$ removal by $\mathrm{CP}$ counters the disabled microvessels [42]. Titers of inflammatory cytokines and choroidal proteins, in CSF and blood, present in different degrees in $\mathrm{AD}$ [43]. Activated astrocytes and microglia congregate in $\mathrm{A} \beta$ plaques [44]. The manner in which $\mathrm{CP}$ inflammatory-signaling molecules modify AD pathogenesis is heterogeneous. Acute inflammation may beneficially promote CSF clearance of affected cells and A $\beta$ aggregates, protecting neurons. However, persistently-elevated $\mathrm{CP}-\mathrm{CSF}$ cytokines and sustained activation of microglia adversely affect neurons. An effective $\mathrm{CP}$ will balance the beneficial vs. detrimental effects of CSF cytokine changes in $\mathrm{AD}, \mathrm{FTD}$ and $\mathrm{HuD}$.

Amyloid beta induces cytokine production; and astrocytes activated by $A \beta$, release inflammatory factors that sustain $\mathrm{A} \beta$ production. Clearly the $\mathrm{CP}-\mathrm{CSF}$, using soluble signals and upregulated cellular adhesion factors, appropriately distributes certain $\mathrm{T}$ cell phenotypes to CSF [12]; such leukocyte penetration into CSF helps to control neuroinflammation and $\mathrm{A} \beta$ levels in $\mathrm{AD}$ brain. The dynamic relationship between pro-inflammatory and anti-inflammatory cytokines in CP-CSF impacts neuroinflammation processes and AD pathology.

Peroxisome-proliferator-activated receptor (PPAR) signaling genes, including PPAR $\delta$ and its obligate heterodimer RXR $\alpha$, were enriched in AD CP. PPAR/RXRs are neuroprotective in $A D$ and $A \beta$ therapies due to antiinflammatory and endothelial actions [43]. PPAR activation, through endogenous or synthetic ligands, likely protects $\mathrm{CP}$ by increasing antioxidant capacity and improving energy supply; this maintains fuel for the $\mathrm{Na}$ pump [45] and CSF secretion [46], and increases expression of A $\beta$ transporters [47]. The novel GFT1803 agent (a pan-PPAR agonist that activates all 3 PPAR isoforms) attenuates $A \beta$ loading-induced damage and neuroinflammation [48]. PPAR thus deserves attention as a potential pathway for restoring $\mathrm{CP}-\mathrm{CSF}$ integrity in $\mathrm{AD}$ in order to counter neurodegeneration.

Significant expression differences were also observed between $\mathrm{AD}$ and FTD + HuD. The VEGF signaling pathway (including VEGFA and VEGF receptors FLT1 and FLT4) displayed significant upregulation in AD but not in FTD or HuD. This agrees with our previous findings of increased VEGF within AD CP [1]. VEGF is required for maintaining endothelial cell fenestration in $\mathrm{CP}$ capillaries [49], an important microstructural feature for delivering plasma substances into the choroidal interstitium for epithelial processing.

Cadherin, on the other hand, was upregulated in FTD and $\mathrm{HuD}$ but not AD. Cadherin is a superfamily of cellular adhesion molecules (CAM), that maintain tissue structure and boundaries between cells and organelles. CAM binding also modifies gene expression. Cell-cell adhesions mediate specific immune actions [50], of which there is a plethora in $\mathrm{CP}$ of FTD and $\mathrm{HuD}$ patient specimens. A cadherin family member prominent in $\mathrm{CP}$ is $\gamma$-protocadherin ( $\gamma$-Pcdh), expressed at the apical membrane [51]. Mutation of $\gamma$-Pcdh causes ventricular collapse. Keep et al. proposed an immune and CSF dynamics role for CP $\gamma$-Pcdh [52], that co-expresses with the $\mathrm{NaBCN} 2 \mathrm{Na}$ transporter supporting CSF secretion. This gene may function in CP ion transport-CSF formation by way of apical-microskeletal membrane interactions with $\mathrm{NaBCN} 2$ that regulate ion trafficking [32]. Moreover, Kolmer immune cells, attached to CP apical surface [53], may have an altered function in neurodegenerative diseases when cadherin is upregulated.

We hypothesize that the downregulated expression observed in this study reflects failing metabolic pathways involved in choroid cell and CSF homeostasis. Reduction in methionine-degradation genes is intriguing given that excessive homocysteine, a product of methionine metabolism, is a risk factor for AD [54-57]. Methionine loading increases brain homocysteine, $A \beta$ and phosphotau in mouse models [58]. Decreased expression in AD $\mathrm{CP}$ of the methionine-degrading gene may relate to elevated homocysteine levels in CSF [59]. The impact of augmented CSF homocysteine on raising brain $A \beta$ and tau hints that additional methionine gene studies on $\mathrm{CP}$ transcription factors and metabolism in neurodegenerative diseases are needed.

We also determined a decreased expression of protein translation genes, including multiple eukaryotic translation initiation factors (EIF genes) and ribosomal proteins. $\mathrm{CP}$ has a major role in producing and secreting CSF proteins, e.g., transthyretin that stabilizes $A \beta$ conformation. In $\mathrm{AD}$ there is decreased choroidal synthesis of transthyretin [24], lowering its CSF concentration [60]. Moreover, the heat stress glucose regulatory proteins 78 and 94 in human AD CP are diminished [39], implicating suboptimal glucose or calcium homeostasis. Altered heat stress proteins at the AD BCSFB deserve examination for impact on cerebral metabolism.

Expression of mTOR associates with controlling cell growth and proliferation [61], possibly a factor as damaged choroid epithelial cells need replacement. Our finding of increased fatty acid oxidation and upstream 
mTOR signaling (Fig. 2), with juxtaposed downregulated protein translation, fits existing concepts suggesting altered energy metabolism in $\mathrm{AD}$ onset and progression. While increased PPAR activity downstream of mTOR fits the compensatory adaptation to retain $\mathrm{CP}$ resiliency, there is a disconnect between upregulated mTOR and the downregulated protein translation machinery typically induced by mTOR. This suggests a break in normal mTOR signaling (see Fig. 2) that could undermine $C P$ function or resiliency when challenged with neurodegeneration. This is significant because of CP's pivotal role in providing brain with supportive factors and immune cells that migrate across BCSFB into CSF-brain. Studies need to assess the role of the dynamic $\mathrm{CP}$ transcriptome in providing resiliency to the $\mathrm{BCSFB}$, in order to retain CSF homeostatic reserve for staving off neurodegeneration.

\section{Conclusions}

The AD transcript findings reported herein for bulk CP tissue compare favorably with and expand prior analysis in laser-captured epithelial cells [26]. Such concurrence is remarkable given the different tissue sampling, measurement platforms and patient cohorts. Highlights of our investigation include upregulated genes linked to inflammation and interferon neuroimmune homeostasis, as well as to JAK-STAT and mTOR; and downregulated genes for methionine degradation, protein translation and claudin-5 (tight junction). CP is a complex homeostatic tissue. The BCSFB undergoes deleterious, but sometimes functional and adaptive, changes in dementiarelated pathophysiology. The enriched JAK-STAT and mTOR pathways (Fig. 2), respectively, are likely instrumental in promoting adaptive transcriptional responses and epithelial repair/replacement when $\mathrm{CP}$ is harmed by injuries associating with neurodegeneration. Analyzing biological pathway mechanisms expedites specific pharmacologic targeting.

Future transcriptome work with larger cohorts should delineate gene expression by demographic endpoints, Braak staging, $A \beta$ plaque score, disease duration, ApoE genotype, co-morbidity, and other disease characteristics. The transcriptome distinctions here precisely describe CP-CSF function in, and response to, certain neuropathologies: AD vs. FTD vs. HuD. This categorical approach provides crucial knowledge on the BCSFB role in pathogenesis; and hopefully should improve prophylaxis of various neural diseases. The goal: To identify exact $\mathrm{CP}$ targets to exploit when implementing pharmacologic/genetic therapies to alleviate CSF-brain metabolic distortions in dementia.

\section{Additional files}

Additional file 1: Table S1. Sample Annotations and Patient Demographics: 'QC passed'indicates samples passing both RNA and Affymetrix quality control, and used for analysis. APOE; genotype data consist of combinations of the $\varepsilon 2, \varepsilon 3$ and $\varepsilon 4$ alleles, with $\varepsilon 4$ carrying the highest risk for brain injury; Bioanalyzer (BA); RNA Integrity (RIN).

Additional file 2: Table S2. T-test results for choroid plexus RNA from 6 control, 7 Alzheimer's disease, 4 FTD and 3HUD subjects: T-test p-values and expression differences $\left(\log _{10}\right.$ Disease/Ctrl) for each disease type are provided for all 5760 Affymetrix probesets measured. Values for the AD vs. HuD + FTD subject comparison are also provided.

Additional file 3: Table S3. Geneset Annotations: Worksheets contain pathways enriched among genes upregulated in AD vs. Ctrl (S3a); downregulated in $A D$ vs. Ctrl (S3b); upregulated in AD but not in HuD + FTD vs. Ctrl (S3C); and upregulated in HuD + FTD but not AD vs. Ctrl (S3d). Hypergeometric $p$-values ( $p$-value), Bonferroni-corrected $p$-values (E-value), overlaps and input and background set sizes are provided.

Additional file 4: Table S4. The top 10 most upregulated and top 10 most downregulated genes in the AD vs. Ctrl comparison, with $p<0.01$. Multiple probe set data, where available, are included in the comparisons.

\section{Abbreviations}

A : amyloid beta; AD: Alzheimer's disease; APOE: apolipoprotein; BCSFB: blood-CSF barrier; CP: choroid plexus; CSF: cerebrospinal; EIF: eukaryotic translation initiation factors; FDR: false discovery rate; FTD: frontotemporal dementia; HuD: Huntington's disease; JAK-STAT: Janus kinase/signal transducers and activators of transcription; LRP1: low density lipoprotein receptor-related protein 1; mTOR: mechanistic target of rapamycin; PMI: post-mortem interval; PPAR: peroxisome-proliferator-activate receptor; RAGE: receptor for advanced glycation end product; TGF $\beta$ : transforming growth factor beta; VEGF: vascular endothelial growth factor.

\section{Authors' contributions}

ES conceived the original research design, wrote/edited a significant part of the paper, and coordinated the manuscript input from various authors. KT conducted the bioinformatics/statistical analyses, constructed figures/ tables, and wrote/edited part of the manuscript. MM processed the choroid plexus specimens, contributed to research design, and organized datasets for uploading to the Gene Expression Omnibus (GEO). EN, AP, EF, DS, LC, LP, AV, $A B, J D, T T, B E$, and GS participated in tissue collection/processing, discussed aspects of experimental design/execution, or helped with manuscript preparation. CJ participated in the project planning, wrote/edited a large part of the paper, and integrated manuscript input from all authors. All authors read and approved the final manuscript.

\section{Author details}

${ }^{1}$ Departments of Neurosurgery and Pathology (Neuropathology Division), Rhode Island Hospital, The Warren Alpert Medical School, Brown University, Providence, RI, USA. ${ }^{2}$ Genetics and Pharmacogenomics, Merck \& Co., Inc., West Point, PA, USA. ${ }^{3}$ United Neuroscience, Dublin, Ireland. ${ }^{4}$ Department of Surgery, University of California San Diego Medical Center, Hillcrest, 212 Dickinson Street, San Diego, CA, USA.

\section{Acknowledgements}

The authors would like to thank the families of patients with AD, referred from the Department of Neurology at Rhode Island Hospital, for donating patient brains to the Brown University Brain Tissue for Neurodegenerative Disorders Resource Center.

\section{Competing interests}

The authors declare that they have no competing interests. 


\section{Availability of data and materials}

The datasets analyzed during the current study are available under the Gene Expression Omnibus (GEO) Accession GSE1 10226 at the National Center for Biotechnology Information (NCBI) at the National Library of Medicine, National Institutes of Health.

\section{Consent for publication}

All authors agree with publication of this manuscript.

\section{Ethics approval and consent to participate}

This research was approved by the Institutional Review Board for Clinical Research at Lifespan, Rhode Island Hospital, Providence, RI.

\section{Funding}

This study was supported by funds provided by Merck \& Co. (for CP tissue transcript analyses and bioinformatical/statistical work), and by the Division of Neuropathology at Rhode Island Hospital (for CP tissue storage/processing and manuscript costs)

\section{Publisher's Note}

Springer Nature remains neutral with regard to jurisdictional claims in published maps and institutional affiliations.

Received: 9 January 2018 Accepted: 11 May 2018

Published online: 31 May 2018

\section{References}

1. Stopa EG, Berzin TM, Kim S, Song P, Kuo-LeBlanc V, Rodriguez-Wolf M, Baird A, Johanson CE. Human choroid plexus growth factors: what are the implications for CSF dynamics in Alzheimer's disease? Exp Neurol. 2001;167(1):40-7.

2. Davson H, Segal M. Physiology of the CSF and blood-brain barriers. Boca Raton: CRC; 1996. p. 822

3. Johanson CE, Stopa E, McMillan PN. The blood-cerebrospinal fluid barrier: structure and functional significance. In: Nag S, editor. The bloodbrain and other neural barriers, vol. 686. New York: Springer; 2011. p. 475.

4. Walter HJ, Berry M, Hill DJ, Cwyfan-Hughes S, Holly JM, Logan A. Distinct sites of insulin-like growth factor (IGF)-II expression and localization in lesioned rat brain: possible roles of IGF binding proteins (IGFBPS) in the mediation of IGF-II activity. Endocrinology. 1999;140(1):520-32.

5. Marques F, Sousa JC, Brito MA, Pahnke J, Santos C, Correia-Neves M, Palha JA. The choroid plexus in health and in disease: dialogues into and out of the brain. Neurobiol Dis. 2017;107:32-40.

6. Strazielle N, Khuth ST, Ghersi-Egea JF. Detoxification systems, passive and specific transport for drugs at the blood-CSF barrier in normal and pathological situations. Adv Drug Deliv Rev. 2004;56(12):1717-40.

7. Spector $R$, Johanson CE. Sustained choroid plexus function in human elderly and Alzheimer's disease patients. Fluids Barriers CNS 2013;10(1):28

8. Balusu S, Brkic M, Libert C, Vandenbroucke RE. The choroid plexus-cerebrospinal fluid interface in Alzheimer's disease: more than just a barrier. Neural Regen Res. 2016;11(4):534-7.

9. Engelhardt B, Sorokin L. The blood-brain and the blood-cerebrospinal fluid barriers: function and dysfunction. Semin Immunopathol. 2009:31(4):497-511

10. Reboldi A, Coisne C, Baumjohann D, Benvenuto F, Bottinelli D, Lira S Uccelli A, Lanzavecchia A, Engelhardt B, Sallusto F. C-C chemokine receptor 6 -regulated entry of $\mathrm{TH}-17$ cells into the CNS through the choroid plexus is required for the initiation of EAE. Nat Immunol. 2009;10(5):514-23.

11. Lauer AN, Tenenbaum T, Schroten $H$, Schwerk $C$. The diverse cellular responses of the choroid plexus during infection of the central nervous system. Am J Physiol. 2017. https://doi.org/10.1152/ajpcell.00137.2017.

12. Schwartz M, Baruch $\mathrm{K}$. The resolution of neuroinflammation in neurodegeneration: leukocyte recruitment via the choroid plexus. EMBO J. 2014:33(1):7-22.
13. Schwartz M, Deczkowska A. Neurological disease as a failure of brainimmune crosstalk: the multiple faces of neuroinflammation. Trends Immunol. 2016;37(10):668-79.

14. Spector R, Johanson CE. Choroid plexus failure in the Kearns-Sayre syndrome. Cerebrospinal Fluid Res. 2010;7(1):14

15. Vercellino M, Votta B, Condello C, Piacentino C, Romagnolo A, Merola A, Capello E, Mancardi GL, Mutani R, Giordana MT, et al. Involvement of the choroid plexus in multiple sclerosis autoimmune inflammation: a neuropathological study. J Neuroimmunol. 2008;199(1-2):133-41.

16. Millward JM, Ariza de Schellenberger A, Berndt D, Hanke-Vela L, Schellenberger E, Waiczies S, Taupitz M, Kobayashi Y, Wagner S, Infante-Duarte C. Application of europium-doped very small iron oxide nanoparticles to visualize neuroinflammation with MRI and fluorescence microscopy. Neuroscience. 2017. https://doi.org/10.1016/j.neuroscience.2017.12.014.

17. Kim S, Hwang Y, Lee D, Webster MJ. Transcriptome sequencing of the choroid plexus in schizophrenia. Transl Psychiatry. 2016;6(11):e964.

18. Marques F, Sousa JC, Coppola G, Falcao AM, Rodrigues AJ, Geschwind DH, Sousa N, Correia-Neves M, Palha JA. Kinetic profile of the transcriptome changes induced in the choroid plexus by peripheral inflammation. J Cereb Blood Flow Metab. 2009:29(5):921-32.

19. Silverberg GD, Huhn S, Jaffe RA, Chang SD, Saul T, Heit G, Von Essen A, Rubenstein E. Downregulation of cerebrospinal fluid production in patients with chronic hydrocephalus. J Neurosurg. 2002;97(6):1271-5.

20. Serot JM, Bene MC, Faure GC. Choroid plexus, aging of the brain, and Alzheimer's disease. Front Biosci. 2003:8:s515-21.

21. Gorlé N, Van Cauwenberghe C, Libert C, Vandenbroucke RE. The effect of aging on brain barriers and the consequences for Alzheimer's disease development. Mamm Genome. 2016;27(7-8):407-20.

22. Johanson C, McMillan P, Tavares R, Spangenberger A, Duncan J, Silverberg $\mathrm{G}$, Stopa E. Homeostatic capabilities of the choroid plexus epithelium in Alzheimer's disease. Cerebrospinal Fluid Res. 2004;1(1):3.

23. Oikonomidi A, Lewczuk P, Kornhuber J, Smulders Y, Linnebank M, Semmler A, Popp J. Homocysteine metabolism is associated with cerebrospinal fluid levels of soluble amyloid precursor protein and amyloid beta. J Neurochem. 2016;139(2):324-32

24. González-Marrero I, Giménez-Llort L, Johanson CE, Carmona-Calero EM Castañeyra-Ruiz L, Brito-Armas JM, Castañeyra-Perdomo A, CastroFuentes R. Choroid plexus dysfunction impairs beta-amyloid clearance in a triple transgenic mouse model of Alzheimer's disease. Front Cell Neurosci. 2015;9:17.

25. Johanson CE, Stopa EG, Daiello L, de la Monte S, Ott B. Disrupted bloodCSF barrier to urea and creatinine in mild cognitive impairment and Alzheimer's disease. J Alzheimer's Dis Parkinsonism 2018;8:2. https://doi. org/10.4172/2161-0460.1000435

26. Bergen AA, Kaing S, ten Brink JB, Gorgels TG, Janssen SF, Bank NB. Gene expression and functional annotation of human choroid plexus epithelium failure in Alzheimer's disease. BMC Genomics. 2015;16:956.

27. López-Romero P, González MA, Callejas S, Dopazo A, Irizarry RA. Processing of Agilent microRNA array data. BMC Res Notes. 2010;3:18.

28. Irizarry RA, Hobbs B, Collin F, Beazer-Barclay YD, Antonellis KJ, Scherf U, Speed TP. Exploration, normalization, and summaries of high density oligonucleotide array probe level data. Biostatistics. 2003;4(2):249-64.

29. Steeland S, Gorlé N, Vandendriessche C, Balusu S, Brkic M, Van Cauwenberghe C, Van Imschoot G, Van Wonterghem E, De Rycke R, Kremer A, et al. Counteracting the effects of TNF receptor-1 has therapeutic potential in Alzheimer's disease. EMBO Mol Med. 2018. https://doi.org/10.15252 lemmm.201708300.

30. Johanson C, Silverberg G, Donahue J, Duncan J, Stopa E. Choroid plexus and CSF in Alzheimer's disease: altered expression and transport of proteins and peptides. London: CRC Press LLC; 2005. p. 307-39.

31. Chalbot S, Zetterberg H, Blennow K, Fladby T, Andreasen N, Grundkelabal I, Iqbal K. Blood-cerebrospinal fluid barrier permeability in Alzheimer's disease. J Alzheimers Dis. 2011;25(3):505-15.

32. Brkic M, Balusu S, Van Wonterghem E, Gorlé N, Benilova I, Kremer A, Van Hove I, Moons L, De Strooper B, Kanazir S, et al. Amyloid $\beta$ oligomers disrupt blood-CSF barrier integrity by activating matrix metalloproteinases. J Neurosci. 2015;35(37):12766-78.

33. Podtelezhnikov AA, Tanis KQ, Nebozhyn M, Ray WJ, Stone DJ, Loboda AP. Molecular insights into the pathogenesis of Alzheimer's disease and its relationship to normal aging. PLoS ONE. 2011;6(12):e29610. 
34. Keren-Shaul H, Spinrad A, Weiner A, Matcovitch-Natan O, Dvir-Szternfeld R, Ulland TK, David E, Baruch K, Lara-Astaiso D, Toth B, et al. A unique microglia type associated with restricting development of Alzheimer's disease. Cell. 2017;169(7):1276-90.

35. Kunis G, Baruch K, Rosenzweig N, Kertser A, Miller O, Berkutzki T, Schwartz M. IFN-gamma-dependent activation of the brain's choroid plexus for CNS immune surveillance and repair. Brain. 2013:136(Pt 11):3427-40.

36. Johanson CE, Duncan JA, Stopa EG, Baird A. Enhanced prospects for drug delivery and brain targeting by the choroid plexus-CSF route. Pharm Res. 2005;22(7):1011-37.

37. Vallieres $L$, Rivest $S$. Regulation of the genes encoding interleukin-6, its receptor, and gp130 in the rat brain in response to the immune activator lipopolysaccharide and the proinflammatory cytokine interleukin-1 beta. J Neurochem. 1997;69(4):1668-83.

38. Mesquita SD, Ferreira AC, Gao F, Coppola G, Geschwind DH, Sousa JC, Correia-Neves M, Sousa N, Palha JA, Marques F. The choroid plexus transcriptome reveals changes in type I and II interferon responses in a mouse model of Alzheimer's disease. Brain Behav Immun. 2015:49:280-92.

39. Anthony SG, Schipper HM, Tavares R, Hovanesian V, Cortez SC, Stopa EG, Johanson CE. Stress protein expression in the Alzheimer-diseased choroid plexus. J Alzheimers Dis. 2003;5(3):171-7.

40. Knuckey NW, Finch P, Palm DE, Primiano MJ, Johanson CE, Flanders KC, Thompson NL. Differential neuronal and astrocytic expression of transforming growth factor beta isoforms in rat hippocampus following transient forebrain ischemia. Brain Res Mol Brain Res. 1996;40(1):1-14.

41. Deane R, Wu Z, Zlokovic BV. RAGE (yin) versus LRP (yang) balance regulates alzheimer amyloid beta-peptide clearance through transport across the blood-brain barrier. Stroke J Cereb Circulation. 2004;35(11 Suppl 1):2628-31

42. Silverberg G, Flaherty-Slone S, Messier A, Soltman S, Miller M, Szmydynger-Chodobska J, Chodobski A, Johanson C. Amyloid transporter expression is altered by aging at the blood-brain barrier and choroid plexus. In: Gordon Research Conference. Tilton: New Hampshire; 2006.

43. Brosseron F, Krauthausen M, Kummer M, Heneka MT. Body fluid cytokine levels in mild cognitive impairment and Alzheimer's disease: a comparative overview. Mol Neurobiol. 2014;50(2):534-44.

44. McDonald CL, Hennessy E, Rubio-Araiz A, Keogh B, McCormack W, McGuirk P, Reilly M, Lynch MA. Inhibiting TLR2 activation attenuates amyloid accumulation and glial activation in a mouse model of Alzheimer's disease. Brain Behav Immun. 2016:58:191-200.

45. Vates TS Jr, Bonting SL, Oppelt WW. Na-K activated adenosine triphosphatase formation of cerebrospinal fluid in the cat. Am J Physiol. 1964;206:1165-72.

46. Johanson CE, Duncan JA 3rd, Klinge PM, Brinker T, Stopa EG, Silverberg GD. Multiplicity of cerebrospinal fluid functions: new challenges in health and disease. Cerebrospinal Fluid Res. 2008;5:10.

47. Pascale CL, Miller MC, Chiu C, Boylan M, Caralopoulos IN, Gonzalez L, Johanson CE, Silverberg GD. Amyloid-beta transporter expression at the blood-CSF barrier is age-dependent. Fluids Barriers CNS. 2011;8:21.
48. Kummer MP, Schwarzenberger R, Sayah-Jeanne S, Dubernet M, Walczak R, Hum DW, Schwartz S, Axt D, Heneka MT. Pan-PPAR modulation effectively protects APP/PS1 mice from amyloid deposition and cognitive deficits. Mol Neurobiol. 2014;51(2):661-71.

49. Maharaj AS, Walshe TE, Saint-Geniez M, Venkatesha S, Maldonado AE, Himes NC, Matharu KS, Karumanchi SA, D'Amore PA. VEGF and TGF-beta are required for the maintenance of the choroid plexus and ependyma. J Exp Med. 2008;205(2):491-501.

50. Turner ML. Cell adhesion molecules: a unifying approach to topographic biology. Biol Rev Camb Philos Soc. 1992;67(3):359-77.

51. Lobas MA, Helsper L, Vernon CG, Schreiner D, Zhang Y, Holtzman MJ, Thedens DR, Weiner JA. Molecular heterogeneity in the choroid plexus epithelium: the 22-member $\gamma$-protocadherin family is differentially expressed, apically localized, and implicated in CSF regulation. J Neurochem. 2012;120(6):913-27.

52. Keep RF, Xiang J, Andjelkovic AV. Where did the ventricles go? J Neurochem. 2012;120(6):851-2.

53. Singhrao SK, Neal JW, Rushmere NK, Morgan BP, Gasque P. Differential expression of individual complement regulators in the brain and choroid plexus. Lab Invest J Tech Methods Pathol. 1999:79(10):1247-59.

54. Clarke R, Smith AD, Jobst KA, Refsum H, Sutton L, Ueland PM. Folate, vitamin B12, and serum total homocysteine levels in confirmed Alzheimer disease. Arch Neurol. 1998;55(11):1449-55.

55. McCaddon A, Davies G, Hudson P, Tandy S, Cattell H. Total serum homocysteine in senile dementia of Alzheimer type. Int J Geriatr Psychiatry. 1998;13(4):235-9.

56. Seshadri S, Beiser A, Selhub J, Jacques PF, Rosenberg IH, D'Agostino RB, Wilson PW, Wolf PA. Plasma homocysteine as a risk factor for dementia and Alzheimer's disease. N Engl J Med. 2002;346(7):476-83.

57. Van Dam F, Van Gool WA. Hyperhomocysteinemia and Alzheimer's disease: a systematic review. Arch Gerontol Geriatr. 2009:48(3):425-30.

58. McCampbell A, Wessner K, Marlatt MW, Wolffe C, Toolan D, Podtelezhnikov A, Yeh S, Zhang R, Szczerba P, Tanis KQ, et al. Induction of Alzheimer's-like changes in brain of mice expressing mutant APP fed excess methionine. J Neurochem. 2011;116(1):82-92.

59. van Wijk N, Slot RER, Duits FH, Strik M, Biesheuvel E, Sijben JWC, Blankenstein MA, Bierau J, van der Flier WM, Scheltens $P$, et al. Nutrients required for phospholipid synthesis are lower in blood and cerebrospinal fluid in mild cognitive impairment and Alzheimer's disease dementia. Alzheimers Dement (Amst). 2017:8:139-46.

60. Hansson SF, Andréasson U, Wall M, Skoog I, Andreasen N, Wallin A, Zetterberg $\mathrm{H}$, Blennow K. Reduced levels of amyloid-beta-binding proteins in cerebrospinal fluid from Alzheimer's disease patients. J Alzheimers Dis. 2009;16(2):389-97.

61. Lee H. Phosphorylated mTOR expression profiles in human normal and carcinoma tissues. Dis Markers. 2017:2017:1397063.

Ready to submit your research? Choose BMC and benefit from

- fast, convenient online submission

- thorough peer review by experienced researchers in your field

- rapid publication on acceptance

- support for research data, including large and complex data types

- gold Open Access which fosters wider collaboration and increased citations

- maximum visibility for your research: over 100M website views per year

At BMC, research is always in progress.

Learn more biomedcentral.com/submissions 